\title{
On the ionizing populations in GEHRs: unveiling integrated properties from the analysis of the Wolf-Rayet population
}

\author{
Marcelo Castellanos, Ángeles I. Díaz \\ Departamento de Física Teórica, C-XI, Universidad Autónoma de \\ Madrid, Cantoblanco, E-28049 Madrid, España \\ Elena Terlevich \\ Instituto Nacional de Astrofísica, Óptica y Electrónica, \\ Luis Enrique Erro 1, Tonantzintla, Puebla 72840, México
}

\begin{abstract}
In recent years, the detection of Wolf-Rayet stars in Giant Extragalactic HII Regions (GEHRs) has yielded several questions about our current understanding of massive stars evolution and hot expanding atmospheres, the age of the ionizing populations and their impact onto the physical properties of GEHRs. Here, we present spectrophotometric observations of four extragalactic GEHRs which show WR features in their spectra. Our goal is to reproduce simultaneously the observed WR properties and the emission-line spectra with the help of current evolutionary synthesis models.
\end{abstract}

\section{Wolf-Rayet populations}

Schaerer \& Vacca (1998, SV98) provide accurate predictions, as a function of the cluster age, for the total number of WR stars and their subtype distribution, the broad stellar emission lines and the luminosities and equivalent widths of the two 'WR bumps' at $\lambda 4650 \AA$ and $\lambda 5808 \AA$. We have used these models to derive the age of the ionizing population, which contains WR stars and whose metallicity has been previously derived by means of the calculated ionic temperatures (Castellanos, Díaz \& Terlevich 2002). In three of the analyzed regions, observations and predictions are in excellent agreement for a single instantaneous burst. The derived ages for the WR population range between $3.1 \mathrm{Myr}$ (CDT3) and 4.1 Myr (H13 and CDT4). For the metal-rich region CDT1, a composite population with two clusters: a young cluster $(2.4 \mathrm{Myr})$ with WR stars, and an older one (around $7 \mathrm{Myr}$ ), not containing WR stars which provides the continuum luminosity, is able to reproduce the observed WR features and the $\mathrm{H} \beta$ equivalent widths.

\section{Modeling the emission line spectra}

Regarding the integrated spectral energy distribution from the ionizing cluster, the recent models from Leitherer et al. (1999, STARBURST99) provide an almost selfconsistent frame to be used in combination with the WR models de- 
scribed above. Therefore, since both SV98 and STARBURST99 models make use of the same stellar evolution prescriptions, we have assumed that a STARBURST99 model of a cluster of a given metallicity which contains the same WR/O star ratio as SV98 (corresponding to a given age), must provide the spectral energy distribution of the ionizing radiation. In the case of region $\mathrm{H} 13$ in NGC 628, a single star cluster with ages between 4.0 and $4.7 \mathrm{Myr}$ fit all the observations adequately.

For regions CDT3 and CDT4, STARBURST99 model clusters with the same $\mathrm{WR} / \mathrm{O}$ ratio as those given by SV98 are not able to reproduce simultaneously the observed gas properties and the WR features strength. Finally, for region CDT1, we have used a composite population with two ionising clusters of 2.4 and 7.1 Myr of age calculated with the STARBURST 99 code in which the youngest of the two provides ten times the number of ionizing photons emitted by the oldest. This cluster contains the same WR/O star ratio as the SV98 model reproducing the observed WR features. The computed photo-ionization model is able to reproduce all the observables adequately.

\section{Conclusions}

The fact that the WR features are adequately reproduced by SV98 models seem to imply that the evolutionary tracks are able to predict the right relative numbers of WR/O stars, and their different subtypes at the derived abundances. These relative numbers, combined with the observed emission-line luminosities of the individual WR stars, and the predicted continuum energy distribution of the ionizing population, predict emission-line intensities and equivalent widths of the WR stars that are in excellent agreement with observations. On the other hand, in two of the analyzed regions (CDT3 and CDT4 in NGC 1232), it is not possible to fit the emission-line spectrum, since the population containing WR stars produces a spectral energy distribution which is too hard to explain the emission of the gas. Blanketed atmospheres for WR stars would make the resulting ionizing spectrum much softer, and the observations should be reproduced adequately. For the high metallicity GEHR CDT1 in NGC 1232, a composite population can explain adequately both the WR features and the emission-line spectrum. Composite populations for $\mathrm{H}$ II regions have been found in previous works by Mayya \& Prabhu (1996) and by Díaz et al. (2000) for disc and circumnuclear objects, respectively, from broad-band and $\mathrm{H} \alpha$ photometry.

\section{References}

Castellanos, M., Díaz, Á.I., Terlevich, E. 2002, MNRAS 329, 315

Díaz, Á.I., Álvarez, M., Terlevich, E., Terlevich, R., Portal, M.S., Aretxaga, I. 2000, MNRAS 311, 120.

Leitherer, C., Schaerer, D., Goldader J.D., et al. 1999, ApJS 123, 3 (STARBURST99)

Mayya, Y.D., Prabhu, T.P. 1996, AJ 111, 1252

Schaerer, D., Vacca, W.D. 1998, ApJ 497, 618 (SV98) 